\title{
Response to Traditional Disease-Modifying Anti-Rheumatic Drugs in Indigent South Africans with Early Rheumatoid Arthritis
}

\author{
Hodkinson $\mathrm{B}^{1}$, Musenge $\mathrm{E}^{2}$, Ally $\mathrm{M}^{3}$, Meyer PWA ${ }^{4}$, Anderson $\mathrm{R}^{4}$, and Tikly $\mathrm{M}^{1}$ \\ ${ }^{I}$ Division of Rheumatology, Department of Medicine, Chris Hani Baragwanath Hospital, University of the \\ Witwatersrand, Johannesburg, South Africa, ${ }^{2}$ Biostatistics and Epidemiology Division, School of Public Health, \\ Faculty of Health Sciences, University of the Witwatersrand, Johannesburg, ${ }^{3}$ Department Internal Medicine, \\ University of Pretoria, Pretoria, ${ }^{4}$ Medical Research Council Unit for Inflammation and Immunity, Department of \\ Immunology, Faculty of Health Sciences, University of Pretoria and NHLS - Tshwane Academic Division, Pretoria, \\ South Africa
}

Correspondence to: $\quad$ Bridget Hodkinson MBBCh, FCP (SA)

Division of Rheumatology,

Department of Medicine

Chris Hani Baragwanath Hospital

PO Box Bertsham 2013

South Africa

Telephone: +27119339377

Facsimile: +27119388738

Email: $\underline{\text { drbridget@gmail.com }}$

\begin{abstract}
Objective: To investigate clinical response to traditional disease modifying anti-rheumatic drugs (DMARDs) in indigent South Africans with early RA. Methods: A cohort of patients with early ( $\leq 2$ years) RA who were DMARD-naïve at inception were prospectively assessed for response to DMARDs using the simplified disease activity index (SDAI) over a 12-month period. Patients with low disease activity (LDA) at 12 months were compared to those with moderate and high disease activity with respect to demographic, clinical, autoantibody and radiographic features. Results: The 171 patients (140 females) had a mean (SD) age of 47.1 (12.4) years, symptom duration of 11.7 (7.1) months and baseline SDAI of 39.4 (16.2). There was a significant overall improvement in the
\end{abstract}


SDAI and its components in the $134(78.4 \%)$ patients who completed the 12 months visit, but only $28.4 \%$ of them achieved LDA. The majority of patients $(91 \%)$ were treated with methotrexate as monotherapy or in combination with chloroquine and/or sulphasalazine. Baseline features that independently predicted a LDA state at 12 months were lower HAQ-DI $(\mathrm{p}=0.023)$ and a higher haemoglobin level $(\mathrm{p}=0.048)$. Receiver operating characteristic curve analysis showed that the 6-months SDAI was better than the baseline SDAI in predicting the 12-month SDAI (area under the curve of 0.69 vs. 0.52 , respectively, $\mathrm{p}=0.008$ ). Conclusion: Less than a third of patients achieved low disease activity at 12 months on traditional DMARDs. Patients who have an inadequate response to traditional DMARDs at 6 months are unlikely to show further improvement on traditional DMARDs at 12 months. These findings underscore the need for better disease control by an aggressive tight control strategy, including intense patient education and biologic therapy.

Keywords: Rheumatoid Arthritis, response to therapy, DMARDs, anaemia, South Africa

Rheumatoid arthritis (RA) is a chronic inflammatory arthritis that occurs worldwide, and is characterized by joint inflammation and destruction. It is now well-established that early intervention with disease modifying anti-rheumatic drugs (DMARDs) to suppress inflammation and achieve a low disease activity state prevents radiographic damage and disability ${ }^{1,2}$. The varying course of the disease from a mild intermittent syndrome to an aggressive systemic disease, coupled with the wide choice of therapies that are now available for RA, make prediction of early response to traditional DMARD therapy a key issue. This may allow treatment to be tailored for the individual patient with $\mathrm{RA}^{3}$.

Health services in sub-Saharan Africa, including South Africa, are overwhelmed by the burden of infectious diseases, but there is mounting evidence that the prevalence of non-communicable diseases is increasing ${ }^{4,5}$. The lower socio-economic groups bear the brunt of these illnesses. Cross sectional and retrospective studies in recent years indicate that RA in Africa is often severe and associated with high levels of functional disability ${ }^{6,7}$.

To date there are no published prospective longitudinal studies of early RA in sub-Saharan Africa. In this resource-constrained setting, characterizing patients who are likely to respond to relatively inexpensive traditional DMARD therapy is important so that aggressive treatment with costly biologics can be reserved for those who clearly need it. We therefore undertook a prospective observational study of early RA in search of clinical, biochemical or radiographic predictors of response to traditional DMARD therapy. This study was approved by the University of the Witwatersrand Committee for Research on Human Subjects. 


\section{PATIENTS AND METHODS}

\section{Patients}

The Gauteng Region Early Arthritis (GREAT) registry is an ongoing multi-dimensional project at two state-funded tertiary hospitals in Gauteng, South Africa that service a predominantly Black African indigent urban and peri-urban population. Consenting patients who met the 1987 revised American College of Rheumatology (ACR) classification criteria for RA ${ }^{8}$, had early disease (defined as symptom duration $\leq 2$ years), and were DMARD-naïve were enrolled between mid-2005 and mid-2008. Standard of care was provided with DMARDs and patients were assessed at routine follow-up visits at 2 to 4-monthly intervals.

\section{Clinical and Serological Assessments for study}

Data for the study was collected at baseline, 6 months and 12 months, independent of routine clinic visits. Demographic features of current employment status, highest level of education and smoking history were obtained. At each visit the 28-joint swollen (SJC) and tender (TJC) counts, pain score, patient global and physician global assessment scores, the health assessment disability index (HAQ-DI) ${ }^{9}$, erythrocyte sedimentation rate (ESR), Creactive protein $(\mathrm{CRP})$, haemoglobin $(\mathrm{Hb})$ level and platelet count were documented. Anaemia was defined as $\mathrm{Hb}<$

12 in females and $<13$ in males ${ }^{10}$. At inception, rheumatoid factor (RF) (composite $\operatorname{IgM}, \operatorname{IgG}, \operatorname{IgA}$ ) was assayed by nephelometry (Siemens Healthcare Diagnostics, USA) and anti-cyclic citrullinated peptide antibodies (aCCP) were measured by a second generation assay (Phadia AB, Sweden). Values of $15 \mathrm{IU} / \mathrm{ml}$ and $10 \mathrm{U} / \mathrm{ml}$, respectively, were considered positive. Response to therapy was defined in terms of the simplified disease activity index (SDAI) as follows: remission $\leq 3.3$, low disease activity $(\mathrm{LDA}) \leq 11.0$, moderate disease activity (MDA) $>11 \leq 26$ and high disease activity (HDA) $>26{ }^{11}$. Based on the 12-month SDAI, patients with LDA ("LDA-12") were compared to those with MDA and HDA ("MDA/HDA-12").

\section{Radiographic assessment}

Plain radiographs of the hands and feet taken at inception were scored for radiographic damage using the modified Larsen method where the maximum obtainable score was $200{ }^{12}$, and patients were classified as having "erosive" disease if one or more marginal erosion was observed.

\section{Medical therapy}

Oral methotrexate (MTX), sulphasalazine (SSZ) and chloroquine sulphate (CQ) were prescribed as either monotherapy or combination therapy, with or without low dose oral prednisone $(\leq 7.5 \mathrm{mg} /$ day $)$. Therapy was 
adjusted by the attending physician, based on the clinical response and adverse effects. No patients were received anti-TNF or other biologic therapy. All patients met with the rheumatology nurse educator for information about the disease and therapy at first visit. Poor adherence to therapy was defined as failing to take DMARD therapy as prescribed for 1 month or longer during the 12 months period.

\section{Statistical analysis}

The Student's t-test was applied to compare continuous variables between groups. In the case of categorical variables, the Pearson's Chi-Square test, or where indicated, the 2-tailed Fishers' Exact test was used. Multivariate analysis was performed using stepwise logistic regression, and any demographic or baseline variable with a p-value $\leq 0.15$ in the univariate analysis were included in the model. Receiver operating characteristic (ROC) curve analysis was performed to assess the predictive value of the baseline and the 6-month SDAI. Stata 10 software (StataCorp, USA) was used. A p value of less than 0.05 was considered significant.

\section{RESULTS}

\section{Baseline features and improvement}

At inception, the 171 patients (140 females) had a mean age of 47.1 years and symptom duration of 11.7 months (range 2-24 months) (Table 1). Only 25.7\% of patients had a symptom duration of less than 6 months. The majority $(55.0 \%)$ of patients were unemployed, and the mean number of school years completed was only 8.9 years (range 0-14 years). No patients received tertiary education. Rheumatoid nodules were observed in a fifth of patients and $\mathrm{RF}$ and aCCP were positive in $83.6 \%$ and $80.1 \%$ of patients respectively. Disease activity was high as reflected by a mean SJC of 10.1, a mean SDAI of 39.4 and moderate to severe functional disability with a mean HAQDI of 1.7. Sixty-two patients (36.3\%) were anaemic. The mean Larsen score was 22.2 and $50.9 \%$ of patients had erosive disease.

At 12 months, data was available for 134 patients (Figure 1). In these patients the mean SDAI was 17.1, a significant improvement from baseline $(\mathrm{p}<0.001) ; 28.4 \%$ of patients achieved LDA (of these $10.4 \%$ of patients were in remission), 54.5\% had MDA and 17.2\% patients had HDA (Table 2). Over the 12 month period, 85 patients (63.4\%) received MTX monotherapy, 37 (27.6\%) were on a combination of MTX and CQ, 8 (6.0\%) on SSZ and 4 (3.0\%) received CQ monotherapy. The starting dose of MTX was between 7.5 and $15 \mathrm{mg} /$ week (mean (SD) dose 13.1 (3.2) $\mathrm{mg} /$ week), and the drug was increased gradually to a maximum of $25 \mathrm{mg} /$ week (mean (SD) dose 20.0 (4.3) $\mathrm{mg} /$ week) at 12 months. Low dose prednisone (5-7.5mg/day) was prescribed in $52.2 \%$ of patients. 
Table 1: Baseline clinical and demographic features of 171 early rheumatoid arthritis patients

Black ethnicity $(\%)$

$152(88.9)$

Females (\%)

$140(81.9)$

Age in years - mean (SD)

$47.1(12.4)$

Symptom duration in months - mean (SD)

$11.7(7.1)$

Smoking history (\%)

$40(23.4)$

Unemployed (\%)

$94(55.0)$

Schooling completed in years - mean (SD)

Rheumatoid nodules (\%)

$34(20.0)$

Swollen joint count - mean (SD)

$10.1(6.4)$

Tender joint count - mean (SD)

$\operatorname{ESR}(\mathrm{mm} / \mathrm{hr})-$ mean $(\mathrm{SD})$

$46.0(30.8)$

CRP (mg/l) - mean (SD)

$30.2(38.7)$

Haemoglobin (g/dl) - mean (SD)

$12.5(1.6)$

Anaemia (\%)

$62(36.3)$

Platelet count $\left(\times 10^{y} / 1\right)-$ mean $(\mathrm{SD})$

$366.8(122.6)$

Simplified Disease Activity Index - mean (SD)

$39.4(16.2)$

Health Assessment Questionnaire Disease Index - mean (SD)

$1.7(0.8)$

modified Larsen score - mean (SD)

$22.2(13.0)$

Erosive disease (\%)

87 ( 50.9)

Rheumatoid factor positive (\%)

$143(83.6)$

Anti-cyclic citrullinated peptide antibody positive (\%)

$137(80.1)$ 


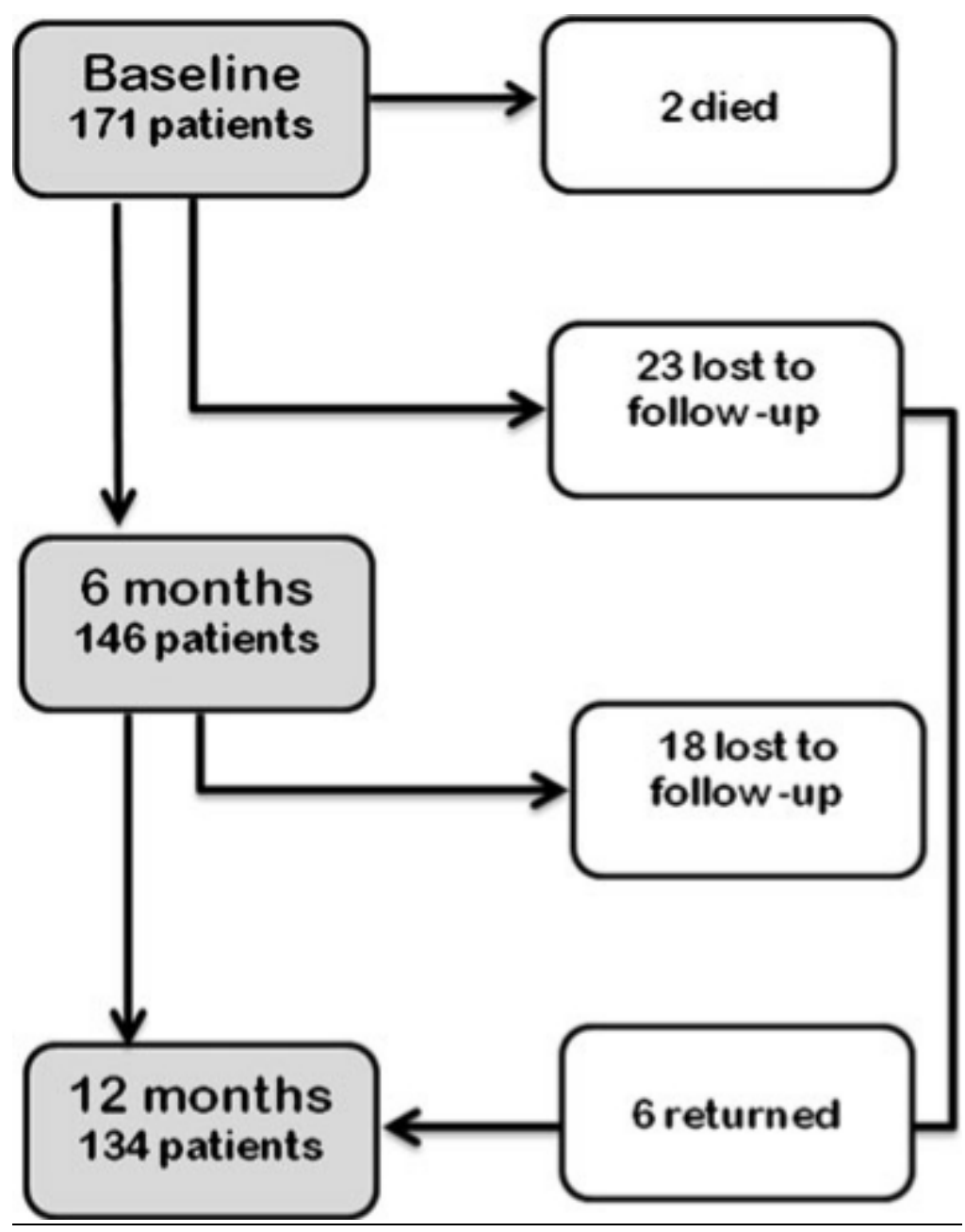

Figure 1: Flow diagram of patients participating in 12 month study

Table 2: Changes in Simplified Disease Activity Index (SDAI) from baseline to 12 months in early RA patients

\begin{tabular}{lccc}
\hline & SDAI category & Baseline & $\mathbf{1 2}$ months \\
& & $\mathrm{n}=171$ & $\mathrm{n}=134$ \\
\hline Remission (\%) & $\leq 3.3$ & 0 & $14(10.4)$ \\
LDA (including remission) (\%) & $\leq 11$ & $4(2.4)$ & $38(28.4)$ \\
MDA (\%) & $>11 \leq 26$ & $37(21.6)$ & $73(54.5)$ \\
HDA (\%) & $>26$ & $130(76.0)$ & $23(17.2)$ \\
\hline
\end{tabular}

LDA: low disease activity, MDA: moderate disease activity, HDA: high disease activity.

Chi-squared test for trend $\mathrm{p}<0.001$. 
Table 3: Clinical, biochemical and radiographic features of 134 early RA patients classified according to SDAI at 12 months - univariate analysis

\begin{tabular}{|c|c|c|c|c|}
\hline & & $\begin{array}{l}\mathrm{LDA}-12 \\
(\mathrm{SDAI} \leq 11) \\
\mathrm{n}=38\end{array}$ & $\begin{array}{l}\mathrm{MDA} / \mathrm{HDA}-12 \\
(\mathrm{SDAI}>11) \\
n=96\end{array}$ & $p$ value \\
\hline \multirow[t]{7}{*}{ Demographics } & Black Ethnicity (\%) & $37(94.4)$ & $83(86.5)$ & $\mathrm{ns}$ \\
\hline & Age (years)-mean (SD) & $43.9(13.0)$ & $48.9(12.3)$ & 0.04 \\
\hline & Female gender $(\%)$ & $29(76.3)$ & $80(83.3)$ & $\mathrm{ns}$ \\
\hline & Symptom duration (months) -mean (SD) & $9.9(6.9)$ & $11.7(6.8)$ & $\mathrm{ns}$ \\
\hline & Smoker (ever) (\%) & $7(18.4)$ & $24(25.0)$ & $\mathrm{ns}$ \\
\hline & Unemployed (\%) & $19(50.0)$ & $54(56.2)$ & $\mathrm{ns}$ \\
\hline & Schooling completed (years)-mean (SD) & $9.8(8.8)$ & $8.6(7.9)$ & 0.06 \\
\hline \multirow{10}{*}{$\begin{array}{l}\text { Baseline Clinical } \\
\text { Assessment }\end{array}$} & SJC -mean (SD) & $9.8(7.3)$ & $10.2(6.1)$ & $\mathrm{ns}$ \\
\hline & TJC -mean (SD) & $12.7(8.4)$ & $14.5(7.5)$ & ns \\
\hline & PAIN (mm) -mean (SD) & $53.4(23.5)$ & $66.7(22.8)$ & 0.006 \\
\hline & PGDA (mm) -mean (SD) & $64.5(25.1)$ & $72.8(24.1)$ & 0.08 \\
\hline & MDGA (mm) -mean (SD) & $52.0(23.5)$ & $60.4(20.1)$ & 0.04 \\
\hline & ESR -mean (SD) & $38.2(25.6)$ & $49.8(29.9)$ & 0.04 \\
\hline & SDAI -mean (SD) & $36.6(18.2)$ & $41.5(15.6)$ & $\mathrm{ns}$ \\
\hline & Haemoglobin (g/dl)- mean (SD) & $13.0(1.5)$ & $11.8(1.6)$ & 0.009 \\
\hline & Platelet count ( x10 $/ 1)$ - mean (SD) & $327.9(93.0)$ & $377.8(115.0)$ & 0.02 \\
\hline & HAQ-DI -mean (SD) & $1.4(0.8)$ & $1.8(0.8)$ & 0.02 \\
\hline \multirow[t]{2}{*}{ Radiology at baseline } & Presence of erosions (\%) & $23(60.5)$ & $50(52.1)$ & ns \\
\hline & modified Larsen score -mean (SD) & $20.8(10.1)$ & $22.4(13.7)$ & $\mathrm{ns}$ \\
\hline \multirow[t]{2}{*}{ Serology } & RF positive (\%) & $33(86.8)$ & $79(82.3)$ & $\mathrm{ns}$ \\
\hline & aCCP positive (\%) & $34(89.5)$ & $78(81.3)$ & ns \\
\hline \multirow[t]{2}{*}{ Therapy } & Poor adherence to treatment (\%) & $9(23.7)$ & $24(25.0)$ & $\mathrm{ns}$ \\
\hline & Corticosteroid prescribed (\%) & $21(55.3)$ & $49(51.0)$ & $\mathrm{ns}$ \\
\hline \multirow{10}{*}{$\begin{array}{l}6 \text { month Clinical } \\
\text { Assessment }\end{array}$} & SJC -mean (SD) & $1.9(2.1)$ & $3.9(4.4)$ & 0.02 \\
\hline & TJC -mean (SD) & $2.8(3.8)$ & $6.5(6.7)$ & 0.005 \\
\hline & PAIN (mm)-mean (SD) & $40.3(24.8)$ & $47.8(25.9)$ & $\mathrm{ns}$ \\
\hline & PGA (mm)-mean (SD) & $38.2(21.10$ & $50.5(26.2)$ & 0.02 \\
\hline & MDGA (mm)-mean (SD) & $20.0(19.9)$ & $41.0(26.6)$ & $<0.001$ \\
\hline & ESR -mean (SD) & $26.9(16.6)$ & $35.2(26.2)$ & 0.08 \\
\hline & SDAI -mean (SD) & $11.7(8.5)$ & $21.1(14.0)$ & 0.001 \\
\hline & Haemoglobin (g/dl)- mean (SD) & $13.8(1.3)$ & $12.6(2.0)$ & 0.007 \\
\hline & Platelet count $\left(\times 10^{9} / 1\right)-$ mean $(\mathrm{SD})$ & $317.8(65.6)$ & $337.2(102.1)$ & 0.02 \\
\hline & HAQ-DI -mean (SD) & $0.6(0.3)$ & $0.8(0.4)$ & 0.01 \\
\hline
\end{tabular}

LDA-12: low disease activity at 12 months, MDA/HAD-12: moderate or high disease activity at 12 months, SDAI: Simplified Disease Activity Index, SJC: 28-joint swollen joint count, TJC: 28-joint tender joint count, PGA: Patient global disease activity assessment, MDGA: Physician global disease activity assessment, ESR: Erythrocyte, Sedimentation Rate, HAQ-DI: Health Assessment Questionnaire Disability Index, RF: Rheumatoid factor, aCCP: Anti-cyclic citrullinated peptide antibody, ns: not significant 
Compared to the MDA/HDA-12 group, the LDA-12 group was significantly younger, had completed more schooling, scored better in baseline pain and physician and patient global scores, and had higher Hb levels and lower platelet counts (Table 3). There was no difference in auto-antibody positivity or titre between the groups, and smokers did not fare worse. Neither the baseline radiographic score or presence of erosions, nor the type of DMARD therapy prescribed nor whether or not the patient received oral corticosteroids were predictive of disease activity at 12 months. Good responses at the 6-months visit of the TJC and SJC, global patient and physician assessments, ESR, SDAI and HAQ-DI were strongly predictive of LDA-12.

Table 4: Multivariate analysis of baseline predictors of low disease activity state at 12 months.

\begin{tabular}{lll}
\hline & OR $(\mathbf{9 5 \%} \mathbf{C I})$ & $\boldsymbol{p}$ value \\
\hline HAQ-DI & $1.89(1.09-3.27)$ & 0.023 \\
Haemoglobin & $0.75(0.56-0.99)$ & 0.048 \\
Age & $1.03(1.00-1.06)$ & 0.072 \\
Platelet count & $1.05(1.00-1.10)$ & 0.070 \\
\hline
\end{tabular}

HAQ-DI- Health Assessment Questionnaire Disability Index

Independent baseline predictors of LDA-12 by multivariate analysis were the HAQ-DI score and $\mathrm{Hb}$, with age and platelet count showing a trend that did not reach statistical significance (Table 4). ROC curve analysis showed that the area under the curve (AUC) for the 6 months SDAI had significantly better predictive utility than the AUC for the baseline SDAI for LDA-12, (0.69 vs 0.52, $\mathrm{p}=0.008)$ (Figure 2). 


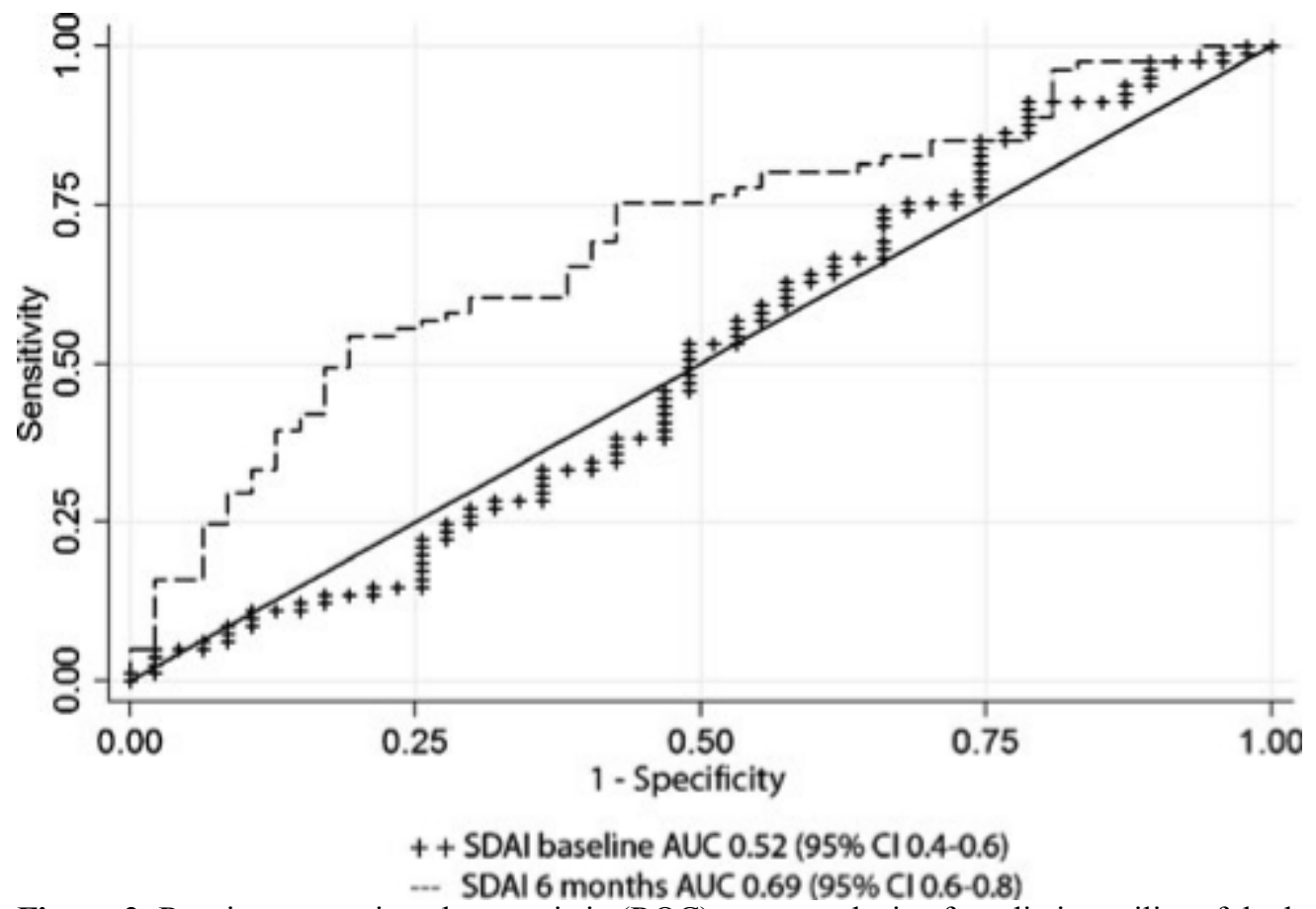

Figure 2: Receiver operating characteristic (ROC) curve analysis of predictive utility of the baseline and 6-month SDAI for achievement of low disease activity state at 12 months. The increasing area under the curve (AUC) corresponds to better predictive utility of the 6-months SDAI compared to the baseline SDAI $(p=0.008)$.

SDAI = Simplified Disease Activity Index

\section{Patients lost to follow-up}

Two patients died within the first 6 months of DMARD therapy of respiratory infections and $35(20.5 \%)$ patients were lost to follow-up over the 12 months period: 17 before the 6 months visit, and a further 18 before the 12 months visit. At baseline, the 'lost-to-follow-up' group differed from those that attended the 12 months visit in that they had a higher mean CRP $(43.3 \mathrm{mg} / \mathrm{dl}$ vs. $26.8 \mathrm{mg} / \mathrm{l}, \mathrm{p}=0.03)$, a trend towards a higher mean SDAI score (42.1 vs. 32.6, $\mathrm{p}=0.06)$ and longer mean disease duration (13.6 months vs 11.2 months, $\mathrm{p}=0.05)$. No significant differences were observed with respect to level of education, employment status, HAQ-DI scores, or radiographic damage. The group of patients who completed the 6 months visit but missed the 12 months visit fared worse at the 6 months visit than the compliant group, with a higher mean SDAI (24.3 vs. 18.1, $\mathrm{p}=0.02)$. Within the group who attended all their study visits, 33 patients $(24.6 \%)$ were deemed to be poorly adherent to therapy, but were otherwise indistinguishable from the rest of the group.

\section{DISCUSSION}

In spite of an overall reduction in disease activity over the 12 months with traditional DMARD therapy in this cohort of indigent patients with early RA, less than a third of patients reached a LDA state, and only $10 \%$ 
achieved remission. Even though our findings are not dissimilar to those reported in a mixed US and Mexican cohort and a Swedish cohort in whom $32 \%$ and $34 \%$ of patients, respectively, achieved a LDA state at 12 months with traditional DMARD treatment ${ }^{13,14}$, the poor response rate in our patients is, at least in part, reflective of their poor socio-economic status. Low levels of formal education and poor socio-economic status have been identified as risk factors for poorer outcome in RA ${ }^{15-17}$. Our patients overall had a low level of education, with those with fewer years of schooling fared worse, and more than half the patients were unemployed at inception. That older age was a poor prognostic factor may be a reflection of the pre-1994 apartheid system in which most Black Africans were deprived of quality education.

Low disease activity and low HAQ-DI scores at baseline are predictive of better SDAI scores at 12 months in the present study. Similar findings have been reported previously in early and established RA where lower joint counts and less functional disability were good prognostic markers for long term low disease activity or remission 18,19. We also observed that higher baseline $\mathrm{Hb}$ level, and a lesser extent lower platelet count, was predictive of a better outcome at 12 months. A recent study supports this finding that RA patients with anaemia have more severe disease $^{20}$. This is most likely on the basis of anaemia of chronic inflammation, mediated by inflammatory cytokines, in particular interleukin (IL)- $6^{21}$. Similarly, a reactive thrombocytosis associated with active RA is thought to be due to the pro-inflammatory effects of IL-1 $\beta$ and IL-4 ${ }^{22,23}$.

The 6-month SDAI was strongly predictive of disease activity at 12 months. The concept that response to DMARDs is evident within 3-6 months of initiating therapy has been described previously ${ }^{24}$ and emphasizes the importance of escalating DMARD therapy early and rapidly in patients who fail to achieve at least LDA. These findings call for a more aggressive approach to the use traditional DMARDs, and an intense "treat-to-target" strategy such as used in the TICORA and FIN-RACo studies, needs to be explored in this setting ${ }^{25,26}$. Notwithstanding the likely gains from a more aggressive approach with traditional DMARDs, judging from experience elsewhere, there will be a need for biologics, despite their cost, for a select group of patients who fail to achieve LDA on traditional DMARDs ${ }^{27}$.

In the present study, neither gender, smoking (past or present) nor auto-antibody status had influence on the outcome, where other studies have reported a more severe disease course in females, smokers, and in those with RF or aCCP ${ }^{28-30}$. The relatively small cohort and short follow-up period might explain our results. Neither baseline radiographic scores nor the presence of erosions were of prognostic importance, but these factors may prove to be of significance when we assess radiological outcome rather than clinical disease activity. 
A disquieting limitation of this study was that about a fifth of patients were lost to follow-up. There is no obvious explanation why this group, which had more active disease at baseline but no significant differences in socio-demographic features compared to the group that completed the 12 months visit, defaulted treatment. It might be a reflection of financial difficulties that preclude these patients from returning for their follow-up visits. It is possible that some of these patients, dissatisfied with their high pain and disability despite commencing treatment at our hospital, may have attended other hospitals or consulted a traditional healer. Another issue of concern is the poor adherence to DMARD therapy in about a quarter of the patients. Both these issues speak to the need for more effective and intense RA education programmes, at both hospital and community levels, to improve outcomes.

It could be argued that symptom duration of 24 months is not "early" disease. This is reinforced by the fact that over half of our patients had erosive disease on plain radiographs at inception. This reflects the patchy and erratic primary care services in many parts of South Africa and underscores the need for training of primary health care workers to recognize and rapidly refer patients with early inflammatory arthritis. A further limitation was the variation in clinical expertise of the attending physicians in assessing disease activity and prescribing DMARDs. This may be an additional reason that better response rates were not achieved.

In summary, a combination of poor socio-economic factors including a low level of formal education, together with older age, high baseline disease activity and functional disability, with a lower haemoglobin level and high platelet count, bodes a poorer prognosis. The study highlights the huge challenge to effectively control disease and reduce disability. The role of tight monitoring and control with both traditional DMARDs and biologics, together with ongoing intense nurse-based patient education need to be explored.

\section{Acknowledgements}

Funding for this study was obtained from the Connective Tissue Diseases Research Fund, University of the Witwatersrand and the Medical Research Council of South Africa.

\section{Conflict of interest}

Hodkinson B: none

Musenge E: none

Ally M: none

Meyer PWA: none

Anderson R: none 


\section{REFERENCES}

1. Smolen JS, Aletaha D, Machold KP. Therapeutic strategies in early rheumatoid arthritis. Best Pract Res Clin Rheumatol 2005;19:163-77.

2. Quinn MA, Cox S. The evidence for early intervention. Rheum Dis Clin North Am 2005;31:575-89.

3. Morel J, Combe B. How to predict prognosis in early rheumatoid arthritis. Best Pract Res Clin Rheumatol 2005;19:137-46.

4. Mayosi BM, Flisher AJ, Lalloo UG, et al. The burden of non-communicable diseases in South Africa. Lancet 2009;374:934-47.

5. Beaglehole R, Bonita R, Horton R, et al. Priority actions for the non-communicable disease crisis. Lancet;377:1438-47.

6. Tikly M, Zannettou N, Hopley M. A longitudinal study of rheumatoid arthritis in South Africans. MedGenMed 2003;5:2.

7. Solomon A, Christian BF, Dessein PH, et al. The need for tighter rheumatoid arthritis control in a South African public health care center. Semin Arthritis Rheum 2005;35:122-31.

8. Arnett FC, Edworthy SM, Bloch DA, et al. The American Rheumatism Association 1987 revised criteria for the classification of rheumatoid arthritis. Arthritis Rheum 1988;31:315-24.

9. Kirwan JR, Reeback JS. Stanford Health Assessment Questionnaire modified to assess disability in British patients with rheumatoid arthritis. Br J Rheumatol 1986;25:206-9.

10. Organisation WH. Nutritional anaemias: Report of a WHO Scientific Group. World Health organ Tech Rep Ser 1968;405:5-37.

11. Aletaha D, Smolen J. The Simplified Disease Activity Index (SDAI) and the Clinical Disease Activity Index (CDAI): a review of their usefulness and validity in rheumatoid arthritis. Clin Exp Rheumatol 2005;23:S100-8.

12. Rau R, Herborn G. A modified version of Larsen's scoring method to assess radiologic changes in rheumatoid arthritis. J Rheumatol 1995;22:1976-82.

13. Saevarsdottir S, Wallin H, Seddighzadeh M, et al. Predictors of response to methotrexate in early DMARD naive rheumatoid arthritis: results from the initial open-label phase of the SWEFOT trial. Ann Rheum Dis. 2011 Mar;70:469-75

14. Khanna D, Oh M, Furst DE, et al. Evaluation of the preliminary definitions of minimal disease activity and remission in an early seropositive rheumatoid arthritis cohort. Arthritis Rheum 2007;57:440-7.

15. Harrison MJ, Tricker KJ, Davies L, et al. The relationship between social deprivation, disease outcome measures, and response to treatment in patients with stable, longstanding rheumatoid arthritis. J Rheumatol 2005;32:2330-6.

16. Vliet Vlieland TP, Buitenhuis NA, van Zeben D, et al. Sociodemographic factors and the outcome of rheumatoid arthritis in young women. Ann Rheum Dis 1994;53:803-6.

17. Sokka T, Kautiainen H, Toloza S, et al. QUEST-RA: quantitative clinical assessment of patients with rheumatoid arthritis seen in standard rheumatology care in 15 countries. Ann Rheum Dis 2007;66:1491-6.

18. Drossaers-Bakker KW, Zwinderman AH, Vliet Vlieland TP, et al. Long-term outcome in rheumatoid arthritis: a simple algorithm of baseline parameters can predict radiographic damage, disability, and disease course at 12-year followup. Arthritis Rheum 2002;47:383-90. 
19. Gossec L, Dougados M, Goupille P, et al. Prognostic factors for remission in early rheumatoid arthritis: a multiparameter prospective study. Ann Rheum Dis 2004;63:67580 .

20. Furst DE, Chang H, Greenberg JD, et al. Prevalence of low hemoglobin levels and associations with other disease parameters in rheumatoid arthritis patients: evidence from the CORRONA registry. Clin Exp Rheumatol 2009;27:560-6.

21. Raj DS. Role of interleukin-6 in the anemia of chronic disease. Semin Arthritis Rheum 2009;38:382-8.

22. Farr M, Scott DL, Constable TJ, et al. Thrombocytosis of active rheumatoid disease. Ann Rheum Dis 1983;42:545-9.

23. Ertenli I, Haznedaroglu IC, Kiraz S, et al. Cytokines affecting megakaryocytopoiesis in rheumatoid arthritis with thrombocytosis. Rheumatol Int 1996;16:5-8.

24. Aletaha D, Funovits J, Keystone EC, et al. Disease activity early in the course of treatment predicts response to therapy after one year in rheumatoid arthritis patients. Arthritis Rheum 2007;56:3226-35.

25. Grigor C, Capell H, Stirling A, et al. Effect of a treatment strategy of tight control for rheumatoid arthritis (the TICORA study): a single-blind randomised controlled trial. Lancet 2004;364:263-9.

26. Rantalaiho V, Korpela M, Laasonen L, et al. Early combination disease-modifying antirheumatic drug therapy and tight disease control improve long-term radiologic outcome in patients with early rheumatoid arthritis: the 11-year results of the Finnish Rheumatoid Arthritis Combination Therapy trial. Arthritis Res Ther;12:R122.

27. van der Kooij SM, de Vries-Bouwstra JK, Goekoop-Ruiterman YP, et al. Limited efficacy of conventional DMARDs after initial methotrexate failure in patients with recent onset rheumatoid arthritis treated according to the disease activity score. Ann Rheum Dis 2007;66:1356-62.

28. van der Helm-van Mil AH, Verpoort KN, Breedveld FC, et al. Antibodies to citrullinated proteins and differences in clinical progression of rheumatoid arthritis. Arthritis Res Ther 2005;7:R949-58.

29. Jawaheer D, Maranian P, Park G, et al. Disease progression and treatment responses in a prospective DMARD-naive seropositive early rheumatoid arthritis cohort: does gender matter? J Rheumatol;37:2475-85.

30. Papadopoulos NG, Alamanos Y, Voulgari PV, et al. Does cigarette smoking influence disease expression, activity and severity in early rheumatoid arthritis patients? Clin Exp Rheumatol 2005;23:861-6. 